\title{
Featured Abstracts
}

The following abstracts were also accepted for display at the meeting

Topic: Atherosclerosis (Clinical), Cardiac Catheterisation

\section{Relationship Between VEGF-C Levels and Mortality in Patients with Peripheral Artery Disease}

\begin{abstract}
Nobutoyo Masunaga, ${ }^{1,2}$ Shuichi Ura, ${ }^{1}$ Mitsuru Ishi, ${ }^{1,2}$ Takashi Unoki, ${ }^{1}$ Daisuke Takagi, ${ }^{2}$ Moritake Iguchi, ${ }^{1,2}$ Kensuke Takabayash, ${ }^{2}$ Yugo Yamashita, ${ }^{2}$ Yasuhiro Hamatani, ${ }^{2}$ Hisashi Ogawa, ${ }^{1,2}$ Noriko Satoh-Asahara, ${ }^{3}$ Akira Shimatsu, ${ }^{3}$ Mitsuru Abe, ${ }^{1,2}$ Masaharu Akao, ${ }^{1,2}$ Koji Hasegawa ${ }^{1}$ and Hiromichi Wada ${ }^{1}$
\end{abstract}

1. Division of Translational Research, Kyoto Medical Center, Kyoto, Japan; 2. Department of Cardiology, Kyoto Medical Center, Kyoto, Japan; 3. Department of Endocrinology, Metabolism and Hypertension, Kyoto Medical Center, Kyoto, Japan; 4. Division of Diabetes Research, National Hospital Organization, Kyoto Medical Center, Kyoto, Japan

Keywords: VEGF-C levels, peripheral artery disease, atherosclerotic disease, all-cause mortality Citation: European Cardiology Review 2018;13(2):123. Dol: https://doi.org/10.15420/ecr.2018.13.2.PO4

The lymphatic system has been suggested to play an important role in cholesterol metabolism and cardiovascular disease. Vascular endothelial growth factor-C (VEGF-C) plays a key role in lymphangiogenesis. Recently, we demonstrated that VEGF-C is closely associated with dyslipidaemia and atherosclerosis. However, the relationship between VEGF-C levels and mortality in patients with atherosclerotic disease is unknown.

We performed a prospective cohort study involving a total of 204 patients with peripheral artery disease. Patients were followed up over 4 years. The outcome was all-cause death. Serum levels of VEGF-C were measured at baseline. Patients were divided into two groups based on median VEGF-C levels. During the follow-up, a total of 53 patients $(26.0 \%$ ) died from any cause. In Kaplan-Meier analysis, the low-VEGF-C group had a significantly higher risk of all-cause death compared with the high-VEGF-C group ( $<<0.001$ by log-rank test). Furthermore, multivariate Cox proportional hazard analysis revealed that the VEGF-C level was significantly and inversely associated with the risk of all-cause death after adjustment for age, sex, traditional risk factors, chronic kidney disease, and the Fontaine stages (adjusted hazard ratio 0.70 for 1 -SD increase; $95 \% \mathrm{Cl}[0.52$ 0.95]; $\mathrm{p}=0.02$ ). In conclusion, a low VEGF-C value was independently associated with the risk of all-cause mortality in patients with peripheral artery disease. 